\title{
The Evolving XUV Absorber in NGC 3516
}

\author{
Smita Mathur, Belinda Wilkes, and Thomas Aldcroft \\ Harvard-Smithsonian Center for Astrophysics, 60 Garden St., \\ Cambridge, $M A$ 02138, USA
}

\begin{abstract}
For NGC 3516 we find that the X-ray warm absorption and the broad UV associated absorption features can be produced by the same absorbing material. We argue that the evolution of the XUV absorber from pre-1992 to 1995 is consistent with expectation for an expanding, outflowing material.
\end{abstract}

\section{NGC 3516: X-ray and UV Observations}

NGC 3516 contains the strongest UV absorption system known in a Seyfert 1 galaxy. This system contains at least two distinct components: a broad $\left(F W H M \approx 2000 \mathrm{~km} \mathrm{~s}^{-1}\right)$ variable component and a narrow $\left(\sim 500 \mathrm{~km} \mathrm{~s}^{-1}\right)$ nonvariable component. Both the broad and the narrow systems contain high as well as low-ionization absorption lines. Recent observations have shown that the broad high-ionization absorption lines have disappeared since about 1992 (Koratkar et al. 1996 and references therein, Kriss et al. 1996)

We analyze a high signal-to-noise $(S / N)$ ROSAT PSPC archival spectrum of NGC 3516 obtained in 1992. The high $S / N$ allows the strong detection of both $\mathrm{O}$ VII and $\mathrm{O}$ VIII edges independently, in spite of the limited spectral resolution of the PSPC. A warm absorber fit to the data shows that the absorber is highly ionized $\left(U=10_{-2.1}^{+2.6}\right)$, and has a large column density $N_{\mathrm{H}} \approx 10^{22} \mathrm{~cm}^{-2}$.

\section{The XUV Absorber}

In several AGN, the X-ray and the UV absorbers were found to be one and the same (the 'XUV' absorbers, Mathur et al. 1994, 1995). Is it also true for NGC 3516 ? The absorption systems in NGC 3516 are clearly complex with multiple components (Kriss et al. 1996). It is the high-ionization, broad absorption system that is most likely to be associated with the X-ray warm absorber. Investigation of this question is tricky, however, because the broad absorption lines have disappeared. Here we argue that the XUV absorption picture is consistent with the presence of a highly ionized X-ray absorber and the current non-detection of $\mathrm{CIV}$ and $\mathrm{NV}$ broad absorption lines (see Fig. 1). The X-ray absorber must have a UV signature showing $O$ VI absorption lines (Fig. 1). Since there were no simultaneous ROSAT and far-UV observations in 1992, this cannot be directly determined. However, we note that in the 1995 HUT observations, O vi doublets are unresolved, but consistent with being broad $\left(F W H M=1076 \pm 146 \mathrm{~km} \mathrm{~s}^{-1}\right.$, Kriss et al. 1996). 


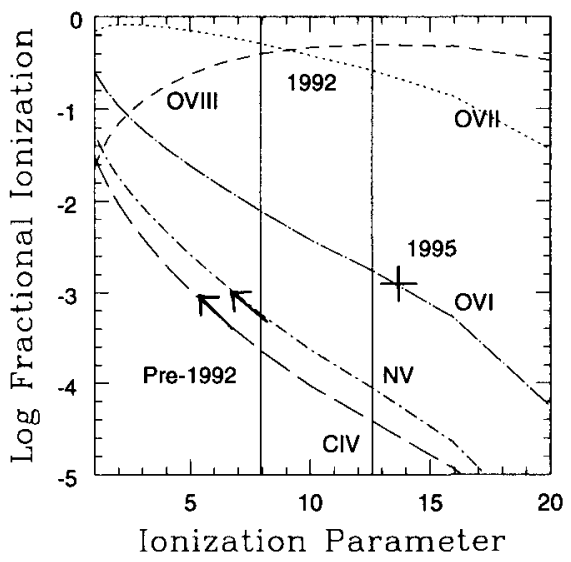

Figure 1. Ionization fractions $f$ of O VI, O viI, O vili, CIV, and $\mathrm{N} v$ as a function of ionization parameter $U$ (with CLOUDY, Ferland 1991). The vertical lines define the range of $U$ for which the ratio $f(\mathrm{O}$ VII $) / f(\mathrm{O}$ VIII $)$ lies within the observed ROSAT range. The arrows on the CIV and $\mathrm{N} v$ curves indicate the lower limits of $f(\mathrm{CIV})$ $\geq 3 \times 10^{-4}$ and $f(\mathrm{Nv}) \geq 3.1 \times 10^{-4}$, based on the published IUE data. The ' + ' mark corresponds to the HUT data in Kriss et al. 1996.

We argue that the XUV absorber in NGC 3516 has evolved with time (Fig. 1). Pre-1992: It showed broad, high-ionization $\mathrm{CIV}$ and $\mathrm{N} \mathrm{V}$ absorption lines and an $\mathrm{X}$-ray ionized absorber $(U \lesssim 7)$. As it evolves, outflowing and expanding, the density falls and the ionization parameter increases. 1992: C IV and $\mathrm{N} V$ absorption lines disappeared; X-ray absorber is still present with $\mathrm{O}$ VI lines in the UV $(U \approx 10)$ (No UV data available to verify). 1995: $\mathrm{C}$ IV and $\mathrm{N} v$ absorption lines remain absent; $\mathrm{X}$-ray absorber is present. O VI lines are present, and detected with $H U T(U \approx 13.5)$. Post-1996: We predict that the O VI absorption lines will disappear as the ionization parameter increases further ( $U \gtrsim 20$ ). The $\mathrm{O}$ VIII edge will continue to strengthen relative to the $\mathrm{O}$ VII edge. Eventually, even the X-ray absorber will also disappear.

Acknowledgments. SM gratefully acknowledges the financial support of NASA grant NAGW-4490 (LTSA) and BW, TA of NASA contract NAS8-39073 (ASC).

\section{References}

Ferland, G.F. 1991 'HAZY', OSU Astronomy Department Internal Report.

Kriss, G., et al. 1996, ApJL, in press.

Koratkar, A., et al. 1996, ApJ, in press.

Mathur, S., Wilkes, B., Elvis, M. \& Fiore, F. 1994, ApJ, 434, 493.

Mathur, S., Elvis, M., \& Wilkes, B. 1995, ApJ, 452, 230. 